\title{
On a Study of Flow Past Non-Newtonian Fluid Bubbles
}

\author{
T. S. L. RADHIKA \\ Department of Mathematics, \\ BITS Pilani, Hyderabad campus \\ Hyderabad, INDIA
}

\author{
T. RAJA RANI \\ Research Fellow, University of Portsmouth, UK. \\ Military Technological College, Muscat \\ OMAN
}

\begin{abstract}
In the current work, we aim at finding an analytical solution to the problem of fluid flow past a pair of separated non-Newtonian fluid bubbles. These bubbles are assumed to be spherical and non-permeable with the non-Newtonian fluid, viz. the couple stress fluid filling their interior. Further, the bubbles are presumed to be static in the flow domain, where a Newtonian fluid streams past these bubbles with a constant velocity (U) along the negative $x$-direction. We developed a mathematical model in the bipolar coordinate system for the fluid flow outside the bubbles and the spherical coordinate system inside the bubbles to derive a separable solution for their respective governing equations. Furthermore, to evaluate the model's applicabilities on the industrial front, the data on some widely used industrial fluids are given as inputs to the model, such as density, the viscosity of air or water for the fluid flow model developed for the region outside the fluid bubbles and the data on Cyclopentane or DIDP (non-Newtonian) for that within the bubbles. Some interesting findings are: the pressure in the outer region of the bubbles is higher when filled with low viscous industrial fluid, Cyclopentane, than a high viscous fluid, DIDP. Furthermore, an increase in the viscosity of Cyclopentane did not alter the pressure distribution in the region outside the bubbles. However, there is a considerable effect on this pressure in the case of DIDP bubbles.
\end{abstract}

Key-Words: - Fluid bubbles, Separated Spheres, Bipolar coordinates, Couple stress fluid, Industrial application, Diisodecyl Phthalate, Cyclopentane.

Received: December 11, 2020. Revised: April 2, 2021. Accepted: April 15, 2021. Published: April 30, 2021.

\section{Introduction}

Translation of fluid sphere in a stationary fluid was first treated, independently, by Rybczynski and Hadamard [1, 2]. They considered the translation of a fluid drop in an immiscible liquid under the action of gravity. The flow was assumed to be axisymmetric, steady, and creeping, and an analytical expression for the stream function was derived. Thus, depicted the streamline patterns. Further, the formula for the drag on a solid sphere given by the Stokes was modified to represent the drag on a fluid bubble. Later, Taylor and Acrivos extended this work and studied the deformation of a falling viscous drop [3]. They showed that for small values of the Weber number "We", the drop deforms into an oblate spheroid, and a further increase deforms it into a spherical cap. Oliver and Chung studied the effect of internal circulation in bubbles and droplets [4]. They evaluated the effects of different density and viscosity ratios on the low Reynolds number flow. They concluded that the drag increases with an increase in the ratio of the bubble's viscosity to the viscosity of ambience. A survey on the related problems indicates works on viscous fluid flow past deformed bubbles and spherical drops partially coated with thin films, as seen in references [5-11]. Ramkissoon, in his work, considered the flow of a Newtonian fluid past a fluid spheroid, the shape of which varies slightly from that of a sphere and derived explicit expressions for external and internal flow fields [5]. Sadhal and Johnson examined the steady axisymmetric creeping flow due to the motion of a liquid drop that is covered partially by a thin immiscible fluid layer [6]. They derived the solution using the perturbation technique and noted a double cell structure's appearance in the circulation inside the film when the primary drop viscosity ratio to that of the bulk fluid is greater than 1/2. Clift et al. [7] and Michaelides [8], in their monographs, presented a critical review of the literature concerning the fluid dynamics, heat and mass transfer of single bubbles. Ramana Murty and Phani Kumar studied the viscous flow past a partially contaminated fluid sphere with a no-slip condition [9]. Dmitry V. Strunin and Adham studied the effect of using two rheological schemes (one with bubbles and the other without) on the wave 
attenuation in the liquid-saturated porous media [10]. Nguyen developed models to study the bubbleparticle encounter mechanics and the bubble-particle sliding interactions[11].

However, to our knowledge, not much work is reported on the flow past fluid bubbles filled with nonNewtonian fluids. Thus, in this paper, we intend to understand the flow configuration and pressure distribution in the flow domain when a viscous (Newtonian) fluid flows past two separated fluid bubbles. Each bubble filled with non-Newtonian fluid. The regions within the fluid bubbles, namely, $S_{i}, i=$ 1,2 are described using the non-Newtonian fluid model proposed by V K Stokes. [12, 13]. The flow in the region outside the bubbles, termed $S_{0}$ is assumed to be governed by the Navier Stokes equation for viscous fluid flow under the Stokesian approximation. We chose the non-conventional system, the bipolar coordinate system, to describe the flow domain [14, 15]. This three-dimensional bipolar coordinate system is an orthogonal coordinate system that results from rotating the two-dimensional bipolar coordinate system about the axis connecting the two foci $F_{1}$ and $F_{2}$. In this system, any point $P$ has coordinates denoted by $(\xi, \eta, \phi)$, where $\xi$ equals the angle $F_{1} P F_{2}$ and $\eta$ equals the natural logarithm of the ratio of distances $d_{1}$ and $d_{2}$ of $P$ from the foci. Now, $\xi=c>0$ where $c$ is constant represents spheres on the positive $x$-axis and $\xi=c<0$ describes spheres on the negative $x$ axis. Thus, with an appropriate choice of the constant " $c$ ", the geometry of separated spheres can be readily constructed, unlike the conventional coordinate systems like Cartesian and Spherical.

Under the assumptions that the fluid is incompressible and flow axisymmetric, the equations governing the Newtonian fluid flow in the region $S_{0}$ takes the form $E^{4} \psi^{(0)}=0$ where $E^{2}$ is the Stokes stream function operator and $\psi^{(0)}$, the stream function in the region $S_{0}$. This equation is separable in the bipolar coordinate system, and hence, an analytical expression for the stream function can be derived. Whereas the flow equations in the region within the fluid bubbles $S_{i}, i=$ 1,2 , in terms of the stream function $\psi^{(i)}, i=1,2$, take the form $E^{4}\left(E^{2}-\alpha^{2}\right) \psi^{(i)}=0$, where, $E^{2}-\alpha^{2}$ is the Helmholtz operator. We know that the Helmholtz operator is not separable in the bipolar coordinate system. As we intended to derive analytical solutions to the developed model, we formulated the fluid flow problem with a spherical coordinate system inside the bubbles. Now, in this system, the Helmholtz equation is separable. Further, we considered two spherical coordinate systems with the origin at the centre of each of the bubbles and solved the Helmholtz equation to determine the stream functions $\psi^{(i)}, i=1,2$ in the two regions $S_{i}, i=1,2$.

Here, the challenge involves implementing the boundary conditions at the interface where we have two different coordinate systems, one on either side. More precisely, we have the expressions of all the flow variables in the region outside the fluid bubbles in the bipolar coordinate system and those within the bubbles in the spherical coordinate system. Hence, we used the coordinate transformation between the spherical and bipolar coordinate systems and represented the flow variables in all the regions in a single system, i.e., the bipolar system. Later, we solved the flow-governing equations together with the boundary conditions for the arbitrary constants. We derived expressions for the flow variables, the stream function and the pressure in all three regions $S_{i}, i=0,1,2$.

Our next objective is to apply this mathematical model to understand the flow of industrial fluids past and within fluid bubbles. Thus, we took widely used industrial (Newtonian) fluids, namely, air or water, to stream past the bubbles and the non-Newtonian fluids Cyclopentane or DIDP fill the bubbles' interior. These two non-Newtonian fluids' choice is due to their vast applications in industries. For instance, Cyclopentane applications include many domestic appliances such as refrigerators and freezers. Moreover, it gets used as a blowing agent in the manufacture of polyurethane insulating foam. Also, it replaces the usage of hazardous gas such as Chloro-Fluoro-Carbons. DIDP, known as a Diisodecyl Phthalate, is used in the polymer industry as a softener and a plasticizer. It can be further used as a lubricant and for manufacturing vinyl and polyvinyl goods such as cable and wire. Its usage further includes manufacturing screen-printing inks, latex coatings, textile coatings, adhesives, sealants, and leather coatings.

\section{Mathematical Formulation of the Problem}

Consider the flow of a Newtonian fluid (with a uniform velocity $U$ at infinity) past a pair of separated fluid spheres fixed in the flow domain, as shown in Fig 1. 


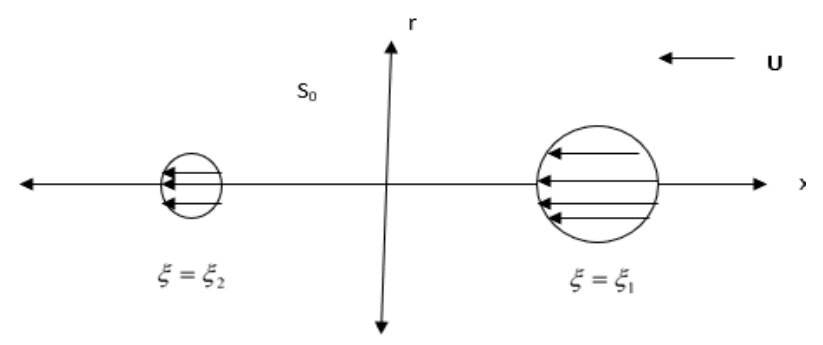

Fig 1. Schematic diagram of flow past separated fluid spheres in bipolar coordinates

The bipolar system represented by the coordinates $(\xi, \eta, \phi)$, with $\left(\hat{e}_{\xi}, \hat{e}_{\eta}, \hat{e}_{\varphi}\right)$ as base vectors $\operatorname{and}\left(h_{\xi}, h_{\eta}, h_{\phi}\right)$ as the corresponding scale factors are to describe the flow domain where

$x=\frac{a \sinh \xi}{\cosh \xi-\cos \eta}$ and $r=\frac{a \sin \eta}{\cosh \xi-\cos \eta}$

$h_{\xi}=\frac{a}{\cosh \xi-\cos \eta} ; h_{\eta}=\frac{a}{\cosh \xi-\cos \eta} ; h_{\phi}=$

$\frac{a \sin \eta}{\cosh \xi-\cos \eta}$

where $-\infty<\xi<\infty, 0 \leq \eta<\pi$.

Here, $\xi=c>0$ where $c$ is constant, it represents spheres on the positive $x$-axis with the centre at a distance $a \operatorname{coth} c$ from the origin (along the $x$-axis) and radius equals $a \operatorname{csch} c . \xi=c<0$ describe spheres on the negative $x$-axis with the centre at a distance $a \operatorname{coth} c$ from the origin (along the $x$-axis) and radius $a \operatorname{csch} c[14,15]$.

Let $\left(\bar{q}^{(i)}, p^{(i)}\right)$ denote the velocity and pressure in the region $S_{i}, i=1,2$ where $S_{1}$ represents the region inside the fluid sphere $\xi=\xi_{1}$ and $S_{2}$, the region inside the fluid sphere $\xi=\xi_{2}$. Let $\left(\bar{q}^{(0)}, p^{(0)}\right)$ be the velocity vector and pressure in the region $S_{0}$ (outside the spheres).

\subsection{Equations governing the fluid flow in the region $S_{0}$}

Assuming that the flow (in the region $S_{0}$ ) is axisymmetric and steady, we have $\bar{q}^{(0)}=$ $u^{(0)}(\xi, \eta) \hat{e}_{\xi}+v^{(0)}(\xi, \eta) \hat{e}_{\eta}$ and the pressure as $p^{(0)}(\xi, \eta)$. Further, considering the fluid to be incompressible, the momentum equations under Stokesian approximation take the form [16]:

$\nabla p^{(0)}+\mu \operatorname{curl}\left(\operatorname{curl} \bar{q}^{(0)}\right)=0$

Now, introducing the stream function $\psi^{(0)}$ through,

$h_{\eta} h_{\phi} u^{(0)}=-\frac{\partial \psi^{(0)}}{\partial \eta} ; h_{\xi} h_{\phi} v^{(0)}=\frac{\partial \psi^{(0)}}{\partial \xi}$

Equation (3) in the bipolar system takes the form, $\frac{1}{h_{\xi}} \frac{\partial p^{(0)}}{\partial \xi}+\frac{\mu}{h_{\eta} h_{\phi}} \frac{\partial}{\partial \eta}\left(E^{2} \psi^{(0)}\right)=0$

$\frac{1}{h_{\eta}} \frac{\partial p^{(0)}}{\partial \eta}-\frac{\mu}{h_{\xi} h_{\varphi}} \frac{\partial}{\partial \xi}\left(E^{2} \psi^{(0)}\right)=0$

where $E^{2}$ is the Stokes stream function operator given by

$E^{2}=\frac{h_{\phi}}{h_{\xi} h_{\eta}}\left\{\frac{\partial}{\partial \xi}\left(\frac{h_{\eta}}{h_{\xi} h_{\phi}} \frac{\partial}{\partial \xi}\right)+\frac{\partial}{\partial \eta}\left(\frac{h_{\xi}}{h_{\eta} h_{\phi}} \frac{\partial}{\partial \eta}\right)\right\}$

Eliminating $p^{(0)}$ from (6) and (7) gives:

$$
E^{4} \psi^{(0)}=0
$$

which is the equation governing the fluid flow in the region $S_{0}$.

\subsection{Equations governing the fluid flow in the} region $S_{i}, i=1,2$

Let $\left(\bar{q}^{(i)}, p^{(i)}\right), i=1,2$ be the velocity vector and pressure in the regions, $S_{i}, i=1,2$, respectively. As discussed earlier, we introduced a spherical coordinate system $(R, \theta, \phi)$ to describe the flow equations in these two regions. Assuming that the flow is axisymmetric and steady, we have $\bar{q}^{(i)}=$ $u^{(i)}(R, \theta) \hat{e}_{r}+v^{(i)}(R, \theta) \hat{e}_{\theta}, i=1,2$ and the pressure as $p^{(i)}(R, \theta), i=1,2$. Further, considering the fluid to be incompressible, the momentum equations under Stokesian approximation take the form:

$\nabla p^{(i)}+\mu \operatorname{curl}\left(\operatorname{curl} \vec{q}^{(i)}+\eta_{1} \operatorname{curl} \operatorname{curl} \operatorname{curl} \vec{q}\right)=0$,

$i=1,2$

where the other symbols in equation (9) have their usual meaning as described in $[10,11]$.

Now, introducing the stream function through

$u^{(i)}=-\frac{1}{R^{2} \sin \theta} \frac{\partial \psi^{(i)}}{\partial \theta} ; v^{(i)}=\frac{1}{R \sin \theta} \frac{\partial \psi^{(i)}}{\partial R}$

equation (9) takes the form:

$\frac{\partial p}{\partial r}+\frac{\mu}{r^{2} \sin \theta} \frac{\partial}{\partial \theta} E^{2} \psi^{(i)}-\frac{\eta_{1}}{r^{2} \sin \theta} \frac{\partial}{\partial \theta} E^{4} \psi^{(i)}=0$

$\frac{1}{r} \frac{\partial p}{\partial \theta}-\frac{\mu}{r \sin \theta} \frac{\partial}{\partial r} E^{2} \psi^{(i)}+\frac{\eta_{1}}{r \sin \theta} \frac{\partial}{\partial r} E^{4} \psi^{(i)}=0$

Now, eliminating pressure from equations (11) and (12), we have

$E^{4}\left(E^{2}-\alpha_{i}{ }^{2}\right) \psi^{(i)}=0, i=1,2, \alpha^{2}=\frac{\mu_{i}}{\eta_{1 i}}$

where $E^{2}=\frac{\partial^{2}}{\partial R^{2}}+\frac{\sin \theta}{R^{2}} \frac{\partial}{\partial \theta}\left(\frac{1}{\sin \theta} \frac{\partial}{\partial \theta}\right)$

and $\mu_{i}, \eta_{1 i}$ are respectively the viscosity and the couple stress momentum parameter in the region $S_{i}, i=1,2$.

\subsection{Boundary conditions}

The determination of the relevant flow field variables $\psi^{(i)}$ and $p^{(i)}, i=0,1,2$ is subject to the following boundary and regularity conditions: 
(i) Continuity of the normal velocity component at interfaces:

$$
u^{(i)}=u^{(0)} \text { on } S_{i}, i=1,2
$$

(ii) Continuity of tangential velocity component at interfaces:

$v^{(i)}=v^{(0)}$ on $S_{i}, i=1,2$

(iii) Continuity of shear stress component at interfaces:

$\tau_{\xi \eta}{ }^{(i)}=\tau_{\xi \eta}{ }^{(0)}$ on $S_{i}, i=1,2$

(iv) Couple stresses vanish at the interface:

$\frac{1}{2} \operatorname{curl} \bar{q}^{(1)}=0$ on $S_{1} ; \frac{1}{2} \operatorname{curl} \bar{q}^{(2)}=0$ on $S_{2}$

$$
\begin{aligned}
& \psi^{(0)}=-\frac{1}{2} U r^{2}, \\
& \text { i.e. } \operatorname{Lim}_{\xi \rightarrow 0} u^{(0)}=-U, \operatorname{Lim}_{\xi \rightarrow 0} v^{(0)}=0 .
\end{aligned}
$$

\section{Solution Methodology}

\subsection{Solution to the equations governing the flow in $S_{0}$}

Using the method of separation of variables, we see after straight forward and lengthy calculations that the solution of equation (8) takes the form $[10,11]$

$$
\begin{gathered}
\psi^{(0)}(\xi, \tau)=a^{2} * \\
(\cosh \xi-\tau)^{-3 / 2} \sum_{n=1}^{\infty}\left(A_{n} \cosh (n-1 / 2) \xi\right. \\
+B_{n} \sinh (n-1 / 2) \xi \\
+C_{n} \cosh (n+3 / 2) \xi \\
\left.+D_{n} \sinh (n+3 / 2) \xi\right) \vartheta_{n+1}(\tau)
\end{gathered}
$$

where $\vartheta_{n+1}(\tau)$ is the Gegenbauer function of order $\mathrm{n}+1$ and degree $-1 / 2, \tau=\cos \eta, \sinh \xi$ and $\cosh \xi$ are hyperbolic trigonometric functions. (v) Regularity condition at infinity, gives:

3.2 Solution to the equations governing the flow in the regions $S_{i}, i=1,2$

From equation (13), using the superposition principle, we see that each $\psi^{(i)}, i=1,2$ can be written as $\psi^{(i)}=$ $\phi_{1}+\phi_{2}, i=1,2$ where

$$
\begin{aligned}
& E^{4} \varphi_{1}=0, \text { and } \\
& \left(E^{2}-\lambda_{i}^{2}\right) \varphi_{2}=0
\end{aligned}
$$

Following Happel and Brenner [14], the stream function in $S_{i}, i=1,2$ is

$$
\begin{aligned}
& \psi^{(i)}(R, \zeta)=\sum_{n=1}^{\infty}\left(A_{n}^{(i)} R^{n+3}+B_{n}^{(i)} R^{n+1}+\right. \\
& \left.C_{n}^{(i)} \sqrt{r} I_{n+1 / 2}\left(\alpha_{i} R\right)\right) \vartheta_{n+1}(\zeta)
\end{aligned}
$$

where $I_{n+1 / 2}(x)$ is the modified Bessel's function of the second kind and $\zeta=\cos \theta$.

\subsection{Determination of arbitrary constants}

As mentioned earlier, at the interface, we have the field variables on either side in two different coordinate systems, namely bi-polar and spherical. Thus, we use the following transformation equations (in their non-dimensional form) to transform all the boundary conditions in the bi-polar system.

$$
R^{2}=\left(\frac{\cosh \xi+\tau}{\cosh \xi-\tau}\right), \theta=\tan ^{-1} \frac{\sqrt{1-\tau^{2}}}{\sinh \xi}
$$

Using (24) and the orthogonality properties of $\vartheta_{n+1}(\tau)$, equations (15)-(18) simplifies to:

(i) Continuity of the normal velocity component at interfaces:

$$
\begin{aligned}
& \int_{-1}^{1} \frac{\sqrt{1-\tau^{2}}}{R^{2} \sin \theta(\cosh \xi-\tau)}\left(A_{n}^{(1)} R^{n+3}+B_{n}^{(1)} R^{n+1}+\right. \\
& \left.C_{n}^{(1)} R^{1 / 2} I_{n+1 / 2}\left(\alpha_{1} R\right)\right) P_{n}(\zeta) d \tau=0 \text { on } \xi=\xi_{1} \\
& \int_{-1}^{1} \frac{\sqrt{1-\tau^{2}}}{R^{2} \sin \theta(\cosh \xi-\tau)}\left(A_{n}^{(1)} R^{n+3}+B_{n}^{(1)} R^{n+1}+\right. \\
& \left.C_{n}^{(1)} R^{1 / 2} I_{n+1 / 2}\left(\alpha_{1} R\right)\right) P_{n}(\zeta) d \tau=0 \text { on } \xi=\xi_{2}
\end{aligned}
$$

(ii) Tangential velocity matches at interfaces:

$$
\begin{aligned}
& v^{(0)}=v^{(1)} \text { on } \xi=\xi_{1} \text { gives: } \\
& \quad \sum_{n=1}^{\infty}\left(A_{n}^{(0)} \cosh \left(n-\frac{1}{2}\right) \xi_{1}+B_{n}^{(0)} \sinh \left(n-\frac{1}{2}\right) \xi_{1}+C_{n}^{(0)} \cosh \left(n+\frac{3}{2}\right) \xi_{1}+D_{n}^{(0)} \sinh (n+\right. \\
& \left.\left.\frac{3}{2}\right) \xi_{1}\right) \frac{-3 /\left.2 \sinh \xi_{1}\left(I_{3}(n-1)-I_{3}(n+1)\right)\right|_{\xi=\xi_{1}}}{2 n+1}+\sum_{n=1}^{\infty}\left(\left(n-\frac{1}{2}\right) A_{n}^{(0)} \sinh \left(n-\frac{1}{2}\right) \xi_{1}+\left(n-\frac{1}{2}\right) B_{n}^{(0)} \cosh \left(n-\frac{1}{2}\right) \xi_{1}+\right. \\
& \left.\left(n+\frac{3}{2}\right) C_{n}^{(0)} \sinh \left(n+\frac{3}{2}\right) \xi_{1}+\left(n+\frac{3}{2}\right) D_{n}^{(0)} \cosh \left(n+\frac{3}{2}\right) \xi_{1}\right) \frac{\left.\left(I_{2}(n-1)-I_{2}(n+1)\right)\right|_{\xi=\xi_{1}}}{2 n+1}= \\
& \int_{-1}^{1} \frac{\sin \eta}{\left.(R \sin \theta)\right|_{\xi=\xi_{2}}\left(\cosh \xi_{1}-\tau\right)^{2}} \frac{\partial \psi^{(1)}}{\partial R} d \tau
\end{aligned}
$$


Furthermore, $v^{(0)}=v^{(2)}$ on $\xi=\xi_{2}$ gives:

$\sum_{n=1}^{\infty}\left(A_{n}^{(0)} \cosh \left(n-\frac{1}{2}\right) \xi_{2}+B_{n}^{(0)} \sinh \left(n-\frac{1}{2}\right) \xi_{2}+C_{n}^{(0)} \cosh \left(n+\frac{3}{2}\right) \xi_{2}+D_{n}^{(0)} \sinh (n+\right.$

$\left.\left.\frac{3}{2}\right) \xi_{2}\right) \frac{-3 /\left.2 \sinh \xi_{2}\left(I_{3}(n-1)-I_{3}(n+1)\right)\right|_{\xi=\xi_{2}}}{2 n+1}+\sum_{n=1}^{\infty}\left(\left(n-\frac{1}{2}\right) A_{n}^{(0)} \sinh \left(n-\frac{1}{2}\right) \xi_{2}+\left(n-\frac{1}{2}\right) B_{n}^{(0)} \cosh \left(n-\frac{1}{2}\right) \xi_{2}+\right.$ $\left.\left(n+\frac{3}{2}\right) C_{n}^{(0)} \sinh \left(n+\frac{3}{2}\right) \xi_{2}+\left(n+\frac{3}{2}\right) D_{n}^{(0)} \cosh \left(n+\frac{3}{2}\right) \xi_{2}\right) \frac{\left.\left(I_{2}(n-1)-I_{2}(n+1)\right)\right|_{\xi=\xi_{2}}}{2 n+1}=$

$\int_{-1}^{1} \frac{\sin \eta}{(R \sin \theta) \mid \xi_{\xi=\xi_{2}}\left(\cosh \xi_{2}-\tau\right)^{2}} \frac{\partial \psi^{(2)}}{\partial R} d \tau$

(iii) Continuity of shear stress component at interfaces:

$$
\tau_{\xi \eta}{ }^{(0)}=\tau_{R \theta}^{(1)} \text { on } \xi=\xi_{1} \text { gives: }
$$

$$
\begin{aligned}
& \left(\left(\begin{array}{c}
-\frac{3}{2} \sinh ^{2} \xi_{1} \sum_{n=1}^{\infty}\left(\begin{array}{c}
A_{n}^{(0)} \cosh \left(n-\frac{1}{2}\right) \xi_{1}+B_{n}^{(0)} \sinh \left(n-\frac{1}{2}\right) \xi_{1} \\
+C_{n}^{(0)} \cosh \left(n+\frac{3}{2}\right) \xi_{1}+D_{n}^{(0)} \sinh \left(n+\frac{3}{2}\right) \xi_{1}
\end{array}\right)\left(\frac{I_{3}(n-1)-I_{3}(n+1)}{2 n+1}\right)_{\xi=\xi_{1}}
\end{array}\right)\right. \\
& -\frac{3}{2} \cosh \xi_{1} \sum_{n=1}^{\infty}\left(\begin{array}{c}
A_{n}^{(0)} \cosh \left(n-\frac{1}{2}\right) \xi_{1}+B_{n}^{(0)} \sinh \left(n-\frac{1}{2}\right) \xi_{1} \\
+C_{n}^{(0)} \cosh \left(n+\frac{3}{2}\right) \xi_{1}+D_{n}^{(0)} \sinh \left(n+\frac{3}{2}\right) \xi_{1}
\end{array}\right)\left(\frac{I_{2}(n-1)-I_{2}(n+1)}{2 n+1}\right)_{\xi=\xi_{1}}+ \\
& \left\{\begin{array}{l}
\sum_{n=1}^{\infty}\left(\begin{array}{c}
A_{n}^{(0)}\left(n-\frac{1}{2}\right)^{2} \cosh \left(n-\frac{1}{2}\right) \xi_{1}+B_{n}^{(0)}\left(n-\frac{1}{2}\right)^{2} \sinh \left(n-\frac{1}{2}\right) \xi_{1} \\
+C_{n}^{(0)}\left(n+\frac{3}{2}\right)^{2} \cosh \left(n+\frac{3}{2}\right) \xi_{1}+D_{n}^{(0)}\left(n+\frac{3}{2}\right)^{2} \sinh \left(n+\frac{3}{2}\right) \xi_{1}
\end{array}\right)\left(\frac{I_{1}(n-1)-I_{1}}{2 n+1}\right. \\
\frac{3}{2} \sum_{n=1}^{\infty}\left(\begin{array}{c}
A_{n}^{(0)} \cosh \left(n-\frac{1}{2}\right) \xi_{1}+B_{n}^{(0)} \sinh \left(n-\frac{1}{2}\right) \xi_{1} \\
+C_{n}^{(0)} \cosh \left(n+\frac{3}{2}\right) \xi_{1}+D_{n}^{(0)} \sinh \left(n+\frac{3}{2}\right) \xi_{1}
\end{array}\right)\left(\frac{I_{5}(n-1)-I_{5}(n+1)}{2 n+1}\right)_{\xi=\xi_{1}}
\end{array}\right. \\
& -\sum_{n=1}^{\infty}\left(\begin{array}{c}
A_{n}^{(0)} \cosh \left(n-\frac{1}{2}\right) \xi_{1}+B_{n}^{(0)} \sinh \left(n-\frac{1}{2}\right) \xi_{1} \\
+C_{n}^{(0)} \cosh \left(n+\frac{3}{2}\right) \xi_{1}+D_{n}^{(0)} \sinh \left(n+\frac{3}{2}\right) \xi_{1}
\end{array}\right)\left(I_{4}(n)\right)_{\xi=\xi_{1}} \\
& =\left.\int_{-1}^{1} \frac{\sqrt{1-\tau^{2}}}{\left(\cosh \xi_{1}-\tau\right)^{3}} \eta_{1}\left(\alpha_{1}^{2}\left(\frac{\partial v^{(1)}}{\partial R}-\frac{v^{(1)}}{R}+\frac{1}{R} \frac{\partial u^{(1)}}{\partial \theta}\right)-\frac{1}{R \sin \theta} E^{4} \psi^{(1)}\right)\right|_{\xi=\xi_{1}} d \tau
\end{aligned}
$$

and $\tau_{\xi \eta}{ }^{(0)}=\tau_{R \theta}^{(2)}$ on $\xi=\xi_{2}$ gives: 


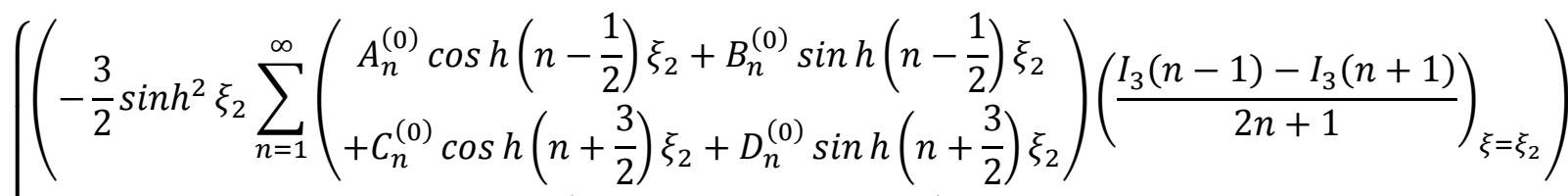

$$
\begin{aligned}
& -\frac{3}{2} \cosh \xi_{2} \sum_{n=1}^{\infty}\left(\begin{array}{c}
A_{n}^{(0)} \cosh \left(n-\frac{1}{2}\right) \xi_{2}+B_{n}^{(0)} \sinh \left(n-\frac{1}{2}\right) \xi_{2} \\
+C_{n}^{(0)} \cosh \left(n+\frac{3}{2}\right) \xi_{2}+D_{n}^{(0)} \sinh \left(n+\frac{3}{2}\right) \xi_{2}
\end{array}\right)\left(\frac{I_{2}(n-1)-I_{2}(n+1)}{2 n+1}\right)_{\xi=\xi_{2}}+ \\
& \left\{\sum_{n=1}^{\infty}\left(\begin{array}{c}
A_{n}^{(0)}\left(n-\frac{1}{2}\right)^{2} \cosh \left(n-\frac{1}{2}\right) \xi_{2}+B_{n}^{(0)}\left(n-\frac{1}{2}\right)^{2} \sinh \left(n-\frac{1}{2}\right) \xi_{2} \\
+C_{n}^{(0)}\left(n+\frac{3}{2}\right)^{2} \cosh \left(n+\frac{3}{2}\right) \xi_{2}+D_{n}^{(0)}\left(n+\frac{3}{2}\right)^{2} \sinh \left(n+\frac{3}{2}\right) \xi_{2}
\end{array}\right)\left(\frac{I_{1}(n-1)-I_{1}(n+1)}{2 n+1}\right)_{\xi=\xi_{2}}+\right\} \\
& \frac{3}{2} \sum_{n=1}^{\infty}\left(\begin{array}{c}
A_{n}^{(0)} \cosh \left(n-\frac{1}{2}\right) \xi_{2}+B_{n}^{(0)} \sinh \left(n-\frac{1}{2}\right) \xi_{2} \\
+C_{n}^{(0)} \cosh \left(n+\frac{3}{2}\right) \xi_{2}+D_{n}^{(0)} \sinh \left(n+\frac{3}{2}\right) \xi_{2}
\end{array}\right)\left(\frac{I_{5}(n-1)-I_{5}(n+1)}{2 n+1}\right)_{\xi=\xi_{2}} \\
& -\sum_{n=1}^{\infty}\left(\begin{array}{c}
A_{n}^{(0)} \cosh \left(n-\frac{1}{2}\right) \xi_{2}+B_{n}^{(0)} \sinh \left(n-\frac{1}{2}\right) \xi_{2} \\
+C_{n}^{(0)} \cosh \left(n+\frac{3}{2}\right) \xi_{2}+D_{n}^{(0)} \sinh \left(n+\frac{3}{2}\right) \xi_{2}
\end{array}\right)\left(I_{4}(n)\right)_{\xi=\xi_{2}} \\
& =\left.\int_{-1}^{1} \frac{\sqrt{1-\tau^{2}}}{\left(\cosh \xi_{2}-\tau\right)^{3}} \eta_{2}\left(\alpha_{2}{ }^{2}\left(\frac{\partial v^{(2)}}{\partial R}-\frac{v^{(2)}}{R}+\frac{1}{R} \frac{\partial u^{(2)}}{\partial \theta}\right)-\frac{1}{R \sin \theta} E^{4} \psi^{(2)}\right)\right|_{\xi=\xi_{2}} d \tau
\end{aligned}
$$

(iv) Couple stresses vanish at the interface:

$$
\begin{aligned}
& \frac{1}{2} \operatorname{curl} \bar{q}^{(1)}=0 \text { on } \xi=\xi_{1} \text { gives } \\
& \left.\quad \int_{-1}^{1} E^{2} \psi^{(1)}\right|_{\xi=\xi_{1}} d \tau=0 \\
& \text { and } \frac{1}{2} \operatorname{curl} \bar{q}^{(2)}=0 \text { on } \xi=\xi_{2} \text { gives } \\
& \left.\quad \int_{-1}^{1} E^{2} \psi^{(2)}\right|_{\xi=\xi_{2}} d \tau=0
\end{aligned}
$$

We see that the above sets of equations to determine the arbitrary constants are infinite series in infinite sets of constants. Solving these equations for the constants is the most crucial and complex task in this study that got handled using the definition of equality of two infinite series that states that " Two infinite series are equal if and only if the corresponding partial sums are equal". Thus, equating the sum to the first one term of the two series (here, we take the right-hand side of the above equations as the zero series) gives ten equations in
$A_{1}^{(0)}, A_{1}^{(1)}, A_{1}^{(2)}, B_{1}^{(0)}, B_{1}^{(1)}, B_{1}^{(2)}, C_{1}^{(0)}, C_{1}^{(1)}, C_{1}^{(2)}, D_{1}^{(0)}$ that is solved for these unknowns. Now, equating the sum to the first two terms of the two series, we again get ten equations in ten unknowns, namely $A_{2}^{(0)}, A_{2}^{(1)}, A_{2}^{(2)}, B_{2}^{(0)}, B_{2}^{(1)}, B_{2}^{(2)}, C_{2}^{(0)}, C_{2}^{(1)}, C_{2}^{(2)}, D_{2}^{(0)}$ that is solved and so on.

\section{Numerical discussions}

As mentioned earlier, in this section, we present the flow configuration and the pressure distribution within and around the fluid bubbles filled with industrial fluids. We considered Newtonian fluids, water or air, to fill the region $S_{0}$ (the region outside the fluid bubbles) and non-Newtonian fluids, Cyclopentane or DIDP in $S_{i}, i=1,2$. Some relevant studies found in [8] and [17]. Data required to carry out the simulations are taken from the published results $[18,19]$ and presented in Table 1 below.

\begin{tabular}{|c|c|c|c|c|}
\hline Fluid & $\begin{array}{c}\text { Temperature } \\
(\mathbf{K})\end{array}$ & $\begin{array}{c}\text { Viscosity } \\
(\mathbf{m P a . s})\end{array}$ & $\begin{array}{c}\text { Temperature } \\
(\mathbf{K})\end{array}$ & $\begin{array}{c}\text { Viscosity } \\
(\mathbf{m P a} . \mathbf{s})\end{array}$ \\
\hline Cyclopentane & 293.15 & 0.443 & 273.15 & 0.563 \\
\hline DIDP & 293.15 & 123 & 283.16 & 267.36 \\
\hline Water & - & 1.002 & - & - \\
\hline Air & - & 0.0018 & - & - \\
\hline
\end{tabular}


Table 1. Data at one atm Pressure

Table 1 shows the values of Cyclopentane's and DIDP's viscosities at one atmospheric pressure and temperatures at $293.15^{\circ} \mathrm{K}, 273.15^{\circ} \mathrm{K}$ and $283.16^{\circ} \mathrm{K}$. These values indicate DIDP as more viscous than compared to Cyclopentane. From the table above, we see that the viscosity of water is higher than that of air.

The values of the couple's stress momentum parameter $" \eta$ " are assigned based on the mathematical constraints derived by Stokes $[12,13]$. For visualizing the flow and pressure plots, we fixed this value inside the region $S_{1}$ as $\eta_{1}=0.01$ and that in the region $S_{2}$ as $\eta_{2}=0.1$. Thus, the couple stress momentum parameter assumes a higher value in the region $S_{2}$ than in $S_{1}$. The viscosity of the fluid within $S_{i}, i=1,2$ is taken to be either $0.443 \mathrm{mPa}$.s in the case of Cyclopentane fluid bubbles or $123 \mathrm{mPa} . \mathrm{s}$ in the case of DIDP bubbles unless specified. Further, the velocity of the fluid at infinity (U) is assumed unity.

Using this data, we solved equations (25) - (32) and (19) to determine the values of the arbitrary constants. We developed codes in MATHEMATICA software to solve the equations and determine the expressions for the stream functions in all three regions $S_{0}, S_{1}$ and $S_{2}$. The expression for the pressure function in the region $S_{0}$ is obtained by eliminating stream function from equations (5) and (6). The resulting equation is solved using the method of separation of variables, the details of which are in Appendix- B.

The pressure function in the regions $S_{i}, i=1,2$ are obtained by integrating equations (11) and (12). After a straight forward calculation, we get the expression for pressure inside the region $S_{i}$ as $P^{(i)}(R, \theta)=\mu_{i} \sum_{n=1}^{\infty} A_{n}^{(i)} \frac{6+4 n}{n} R^{n} P_{n}(\cos \theta), i=1,2$.

The flow pattern and pressure distribution are depicted in Figures 2-15. Figures $2-5$ portray the streamlines and pressure contours when the two bubbles are of equal radius. Figures 6-13 represent the flow configuration and pressure distribution when the two bubbles are of unequal radii.

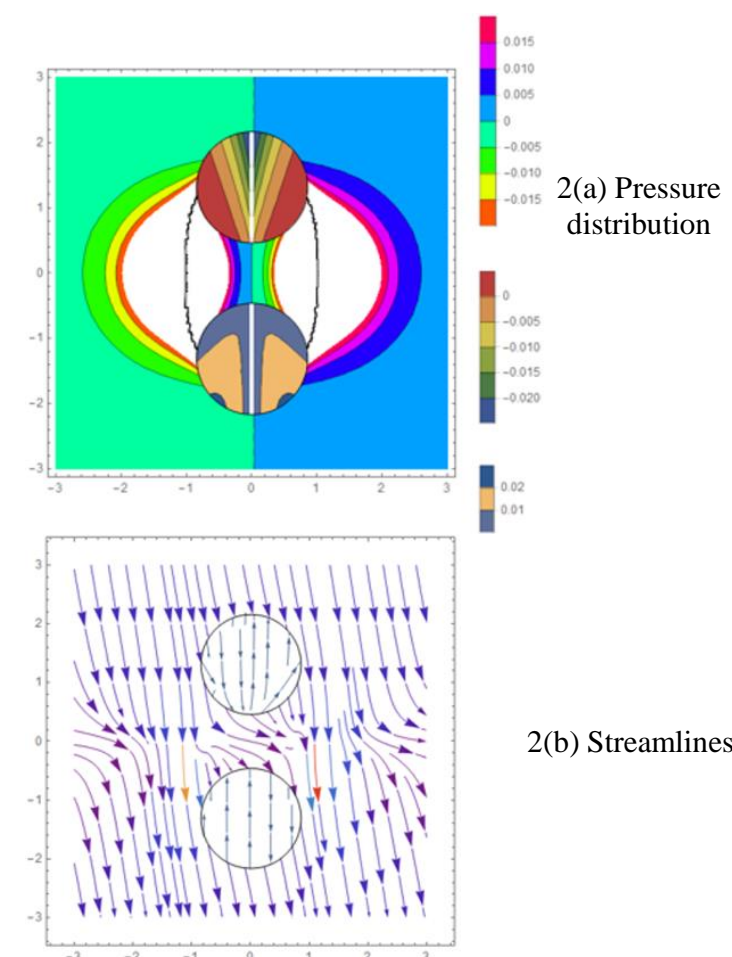

Figure 2. Pressure distribution and streamlines for Cyclopentane fluid bubbles surrounded by water $\xi_{1}=1.0, \xi_{2}=-1.0, \alpha_{1}=0.0664, \alpha_{2}=0.021, \mu_{0}=1.002 \times 10^{-3}$ $\mu_{1}=\mu_{2}=0.4413 \times 10^{-3}$

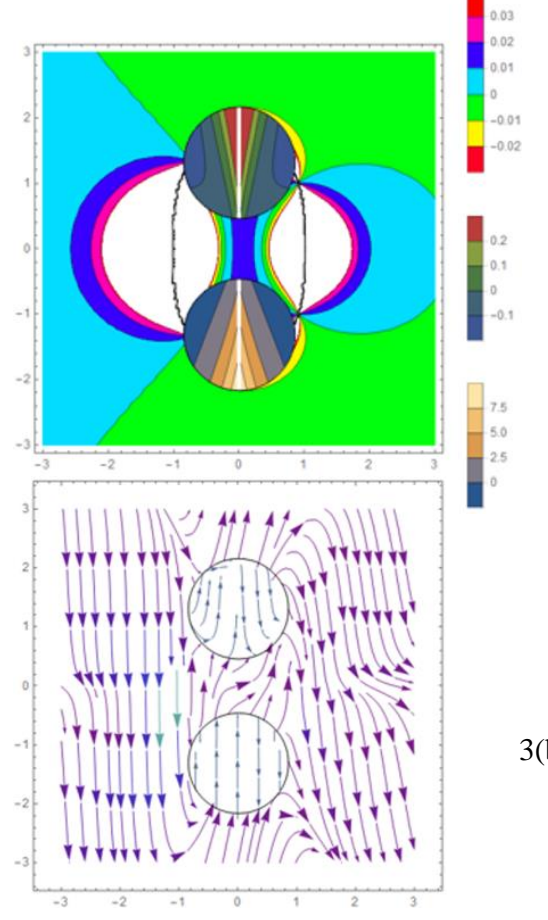

3(a) Pressure distribution

3(b) Streamlines

Figure 3. Pressure distribution and streamlines for DIDP fluid bubbles surrounded by water

$\xi_{1}=1.0, \xi_{2}=-1.0, \alpha_{1}=1.10905, \alpha_{2}=0.350714, \mu_{0}=1.002 \times 10^{-3}$, $\mu_{1}=\mu_{2}=0.123$ 


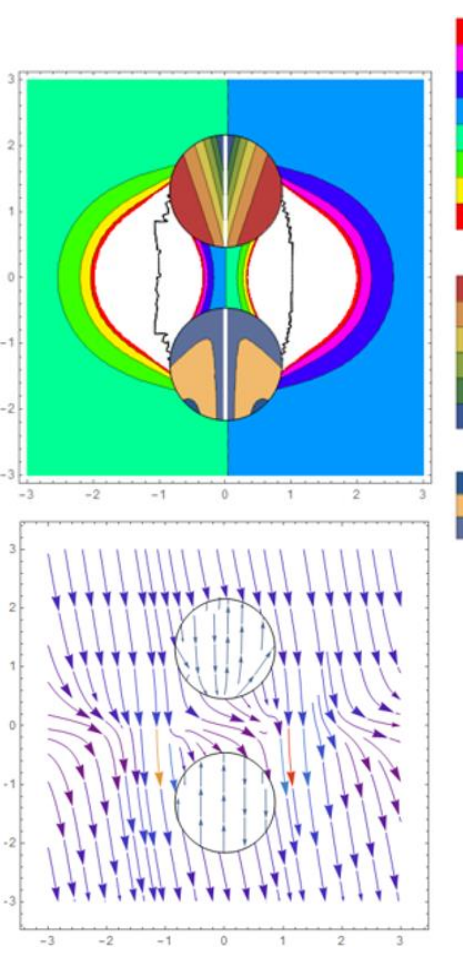

4(a) Pressure distribution

4(b) Streamlines

Figure 4. Pressure distribution and streamlines for Cyclopentane fluid bubbles surrounded by air $\xi_{1}=1.0, \xi_{2}=-1.0, \alpha_{1}=0.0664, \alpha_{2}=0.021, \mu_{0}=1.82 \times 10^{-5}$,
$\mu_{1}=\mu_{2}=0.4413 \times 10^{-3}$
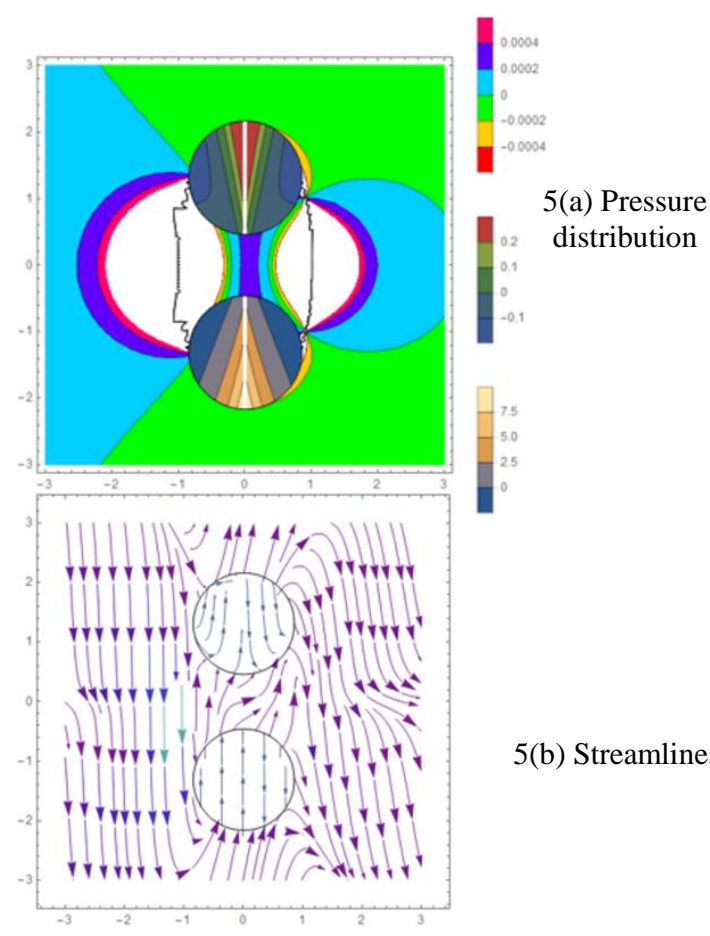

distribution

5(b) Streamlines

Figure 5. Pressure distribution and streamlines for DIDP fluid bubbles surrounded by air

$\xi_{1}=1.0, \xi_{2}=-1.0, \alpha_{1}=1.10905, \alpha_{2}=0.350714, \mu_{0}=1.8 \times 10^{-5}$, $\mu_{1}=\mu_{2}=0.123$

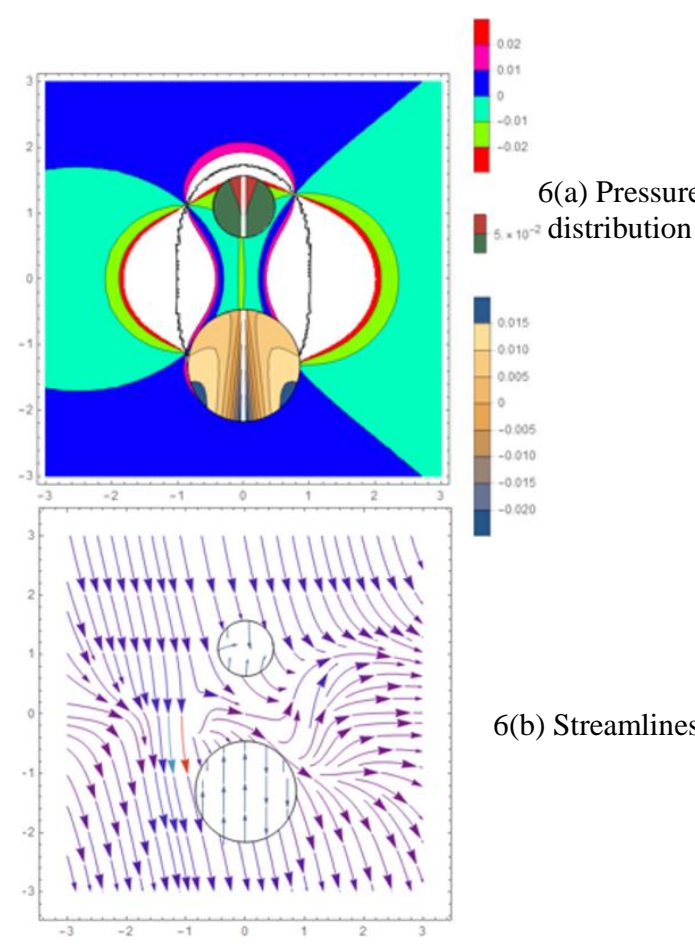

Figure 6. Pressure distribution and streamlines for Cyclopentane fluid bubbles surrounded by water $\begin{aligned} \xi_{1}=1.5, \xi_{2}=-1.0, \alpha_{1} & =0.0664, \alpha_{2}=0.021, \mu_{0}=1.002 \times 10^{-3}, \\ \mu_{1} & =\mu_{2}=0.4413 \times 10^{-3}\end{aligned}$

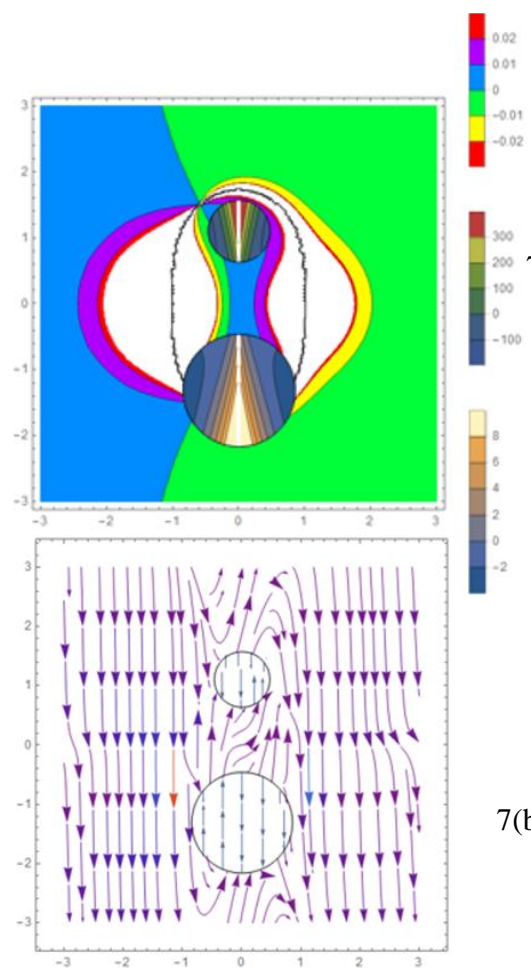

7(a) Pressure distribution

7(b) Streamlines

Figure 7. Pressure distribution and streamlines for DIDP fluid bubbles surrounded by water $\xi_{1}=1.5, \xi_{2}=-1.0, \alpha_{1}=1.10905, \alpha_{2}=0.350714, \mu_{0}=1.002 \times 10^{-3}$ $\mu_{1}=\mu_{2}=0.123$ 


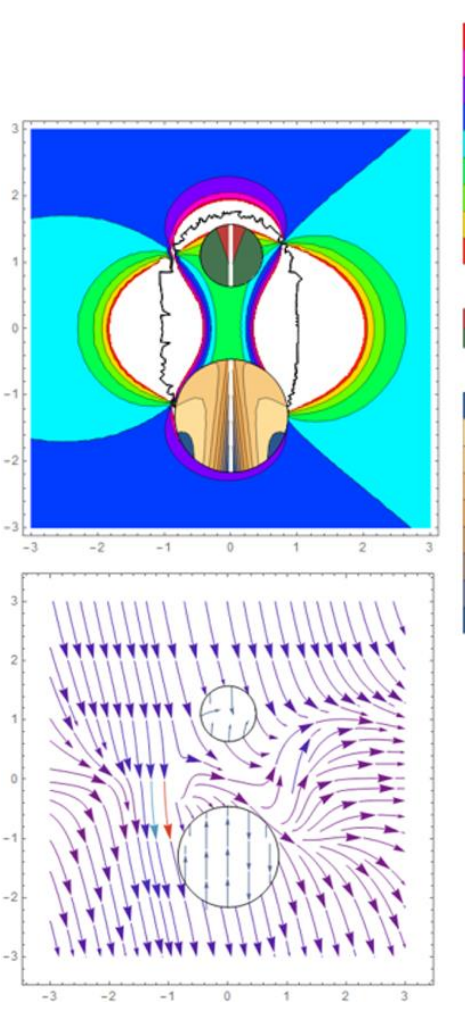

8(b) Streamlines

Figure 8. Pressure distribution and streamlines for Cyclopentane fluid bubbles surrounded by air $\xi_{1}=1.5, \xi_{2}=-1.0, \alpha_{1}=0.0664, \alpha_{2}=0.021, \mu_{0}=1.82 \times 10^{-5}$,
$\mu_{1}=\mu_{2}=0.4413 \times 10^{-3}$

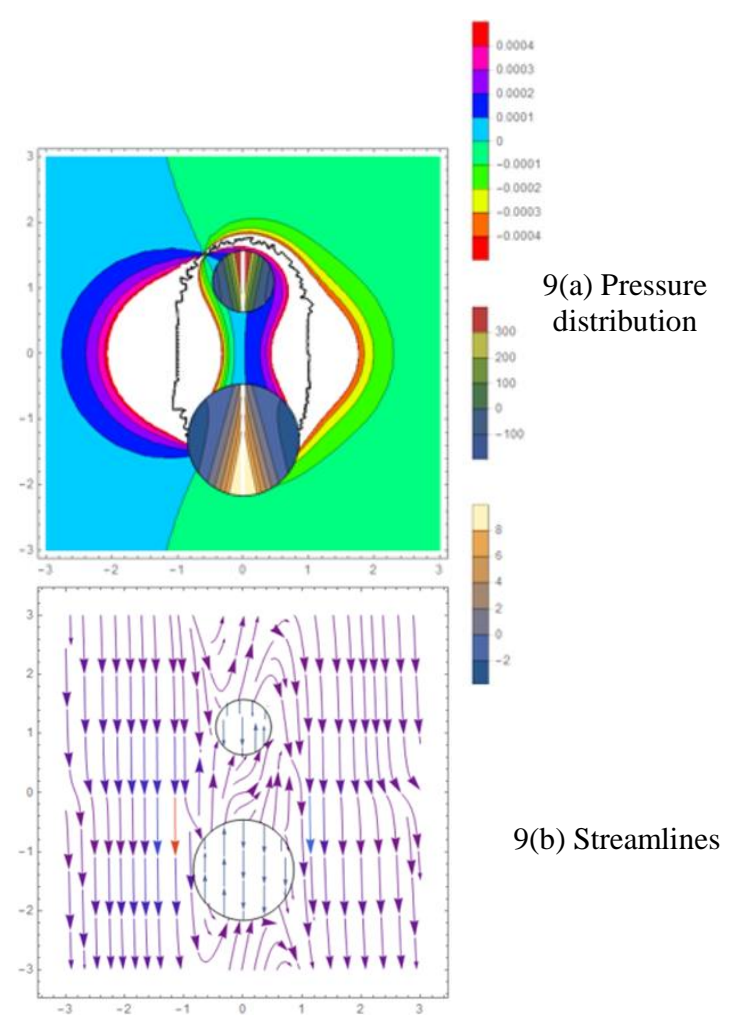

Figure 9. Pressure distribution and streamlines for DIDP fluid bubbles surrounded by air $\begin{aligned} \xi_{1}=1.5, \xi_{2}=-1.0, \alpha_{1}= & 1.10905, \alpha_{2}=0.350714, \mu_{0}=1.8 \times 10^{-5}, \\ & \mu_{1}=\mu_{2}=0.123\end{aligned}$

8(a) Pressure distribution

0.015

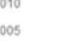

(n) 


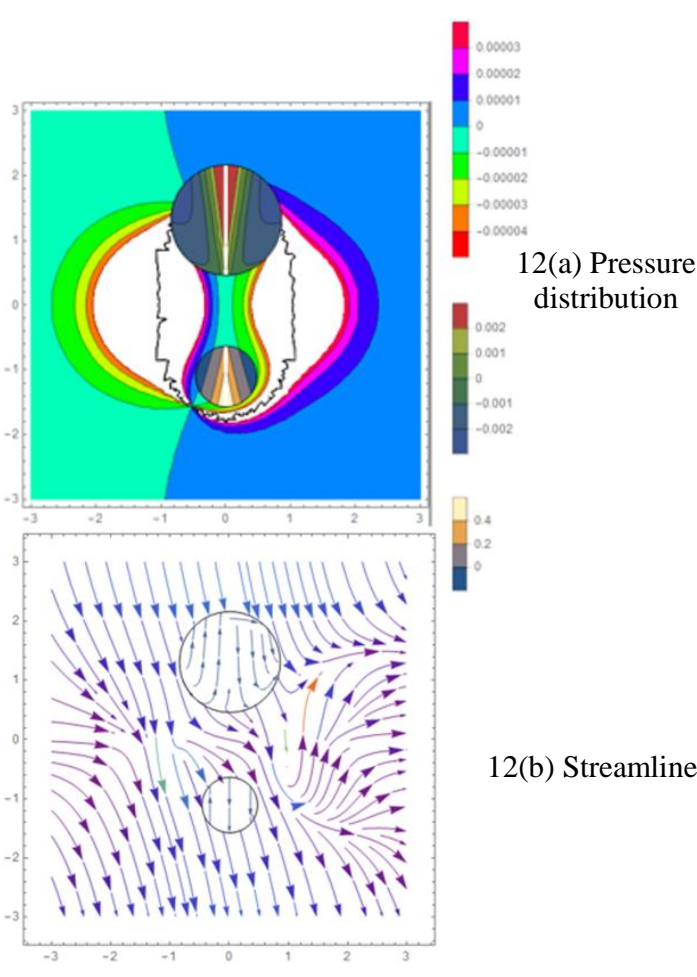

Figure 12. Pressure distribution and streamlines for Cyclopentane fluid bubbles surrounded by air $\xi_{1}=1.0, \xi_{2}=-1.5, \alpha_{1}=0.0664, \alpha_{2}=0.021, \mu_{0}=1.82 \times 10^{-5}$, $\mu_{1}=\mu_{2}=0.4413 \times 10^{-3}$

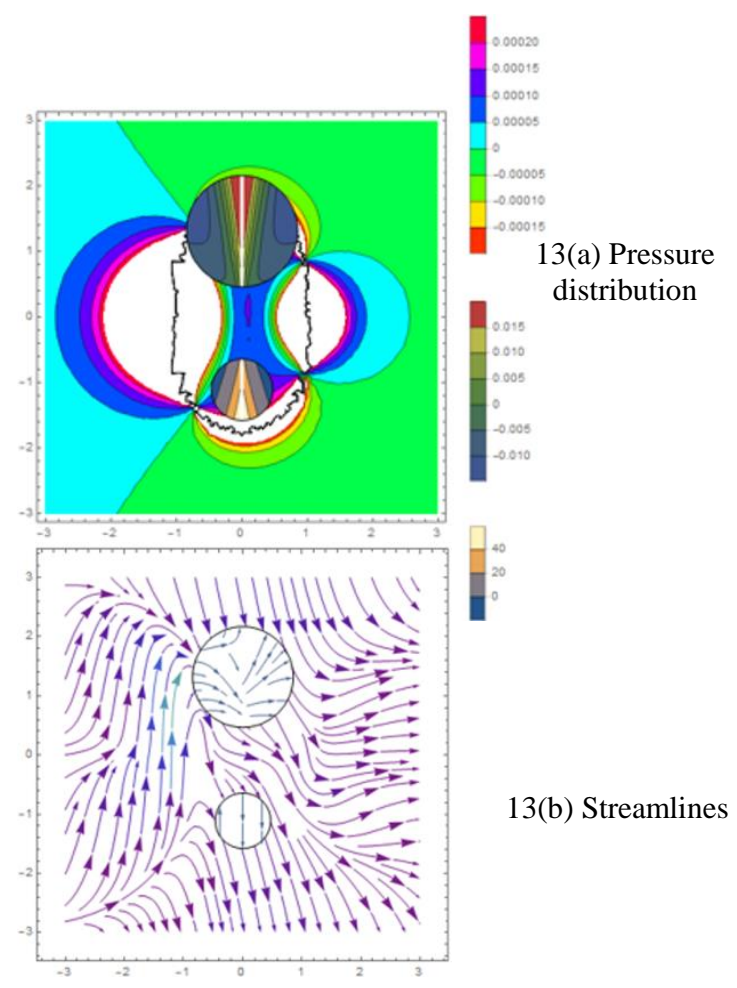

Figure 13. Pressure distribution and streamlines for DIDP fluid bubbles surrounded by air

$\xi_{1}=1.0, \xi_{2}=-1.5, \alpha_{1}=1.10905, \alpha_{2}=0.350714, \mu_{0}=1.8 \times 10^{-5}$, $\mu_{1}=\mu_{2}=0.123$

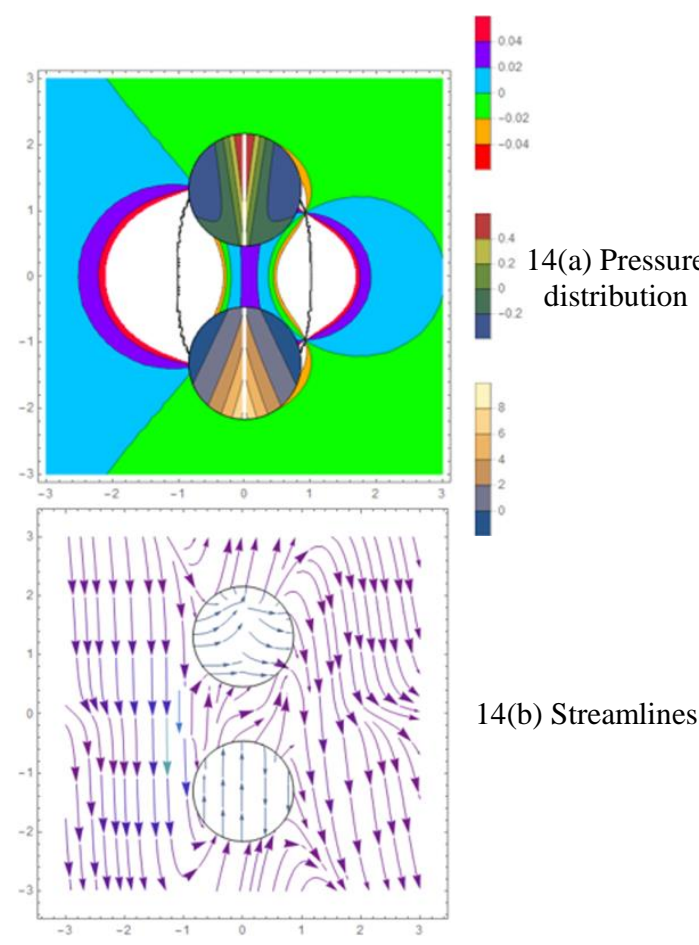

Figure 14. Pressure distribution and streamlines for DIDP fluid bubbles surrounded by water $\xi_{1}=1.0, \xi_{2}=-1.0, \alpha_{1}=1.63401, \alpha_{2}=0.51672, \mu_{0}=1.002 \times 10^{-3}$,
$\mu_{1}=\mu_{2}=0.267$

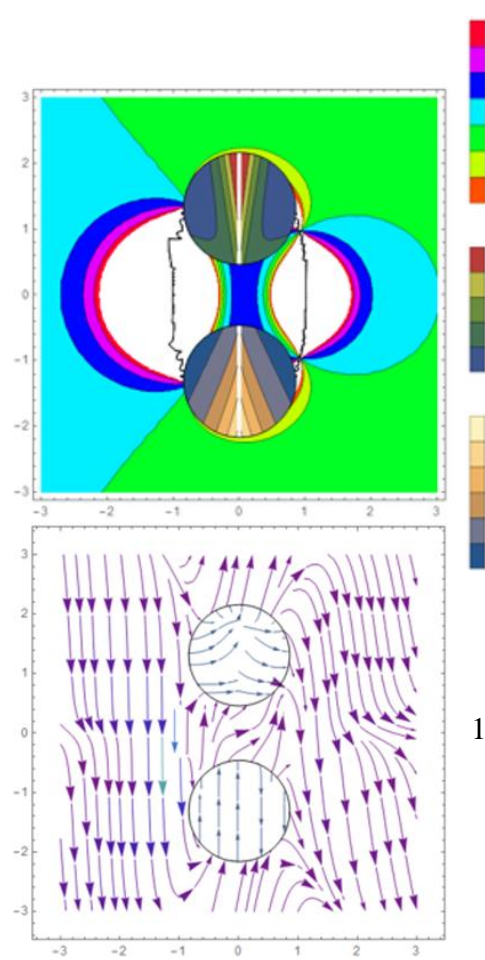

Figure 15. Pressure distribution and streamlines for DIDP fluid bubbles surrounded by air $\xi_{1}=1.0, \xi_{2}=-1.0, \alpha_{1}=1.63401, \alpha_{2}=0.51672, \mu_{0}=1.8 \times 10^{-5}$ $\mu_{1}=\mu_{2}=0.267$ 


\subsection{Observations}

- The pressure around the fluid bubbles is high when surrounded by water than air. As water is a more viscous fluid than air, it offers more resistance to its flow.

- The pressure within DIDP-fluid bubbles is more than that within Cyclopentane bubbles since DIDP is more viscous than Cyclopentane.

- Studies reveal that couple stresses decrease the fluid flow velocity, thereby increasing the pressure in its surroundings [20]. This phenomenon is evident in the case of equal spheres, where the pressure in $S_{1}$ is less than that in $S_{2}$. (te that the couple stress momentum parameter is assigned a lower value for the fluid inside $S_{1}$ than that in $S_{2}$.)

- In the case of Cyclopentane-fluid bubbles, isobaric lines in the region $S_{0}$ are symmetrical about the $x$-axis, whereas they are asymmetrical in the case of DIDP bubbles.

- We see from Figures (3 and 14) and (5 and 15) that, with an increase in the viscosity of DIDP, the pressure in $S_{0}$ took values on a broader scale. However, this phenomenon not being observed in the case of Cyclopentane.

- Figures 14 and 15 present the streamlines and pressure contours with the bubbles filled with the same fluid but with different viscosities. We understand that this difference in the viscosities did not affect the pressure in the region $S_{0}$.

\section{Conclusions}

We developed a mathematical model to simulate a Newtonian fluid flow past a pair of separated nonNewtonian, impermeable fluid bubbles. To evaluate the model's applicabilities on the industrial front, we assigned data on some widely used industrial fluids to the model parameters. Data on the density, viscosity of air or water are the inputs to the fluid flow model outside the fluid bubbles. Whereas the data on Cyclopentane or DIDP is for the parameters for the model within the bubbles. We depicted the flow configuration and the pressure distribution when the fluid bubbles have equal and unequal radii and discussed our observations. From these conclusions, we anticipate a possible means of pressure regulation in transport phenomenon problems from the industrial front; and the prime objective is to regulate pressure in and around spherical objects. This work's future scope is to extend the developed mathematical model to suit some bio-medical engineering applications.

\section{References:}

[1]. Rybczynski W. (1911). Uber die fortschreitende bewegung einer flussigen kugel in einem zahenmedium Bull Acad. Sci. Cracovie, Ser. A, $1,40-46$

[2]. Hadamard J S. (1911). Mouvement permanent lent dune sphere liquid et visqueuse dans u liquid visqueux Compt.

[3]. Taylor TD, Acrivos A. (1964). On the deformation and drag of a falling viscous drop at low Reynolds number, Journal of Fluid Mechanics, 18, 466-476. DOI: $10.1017 / \mathrm{S} 0022112064000349$

[4]. Oliver, D. L. R. and Chung, J. N. (1982). Steady flows inside and around a fluid sphere at low Reynolds numbers, American Institute of Aeronautics and Astronautics Conference

[5]. Ramkissoon, H. (1986). Stokes flow past a slightly deformed fluid sphere. Journal of Applied Mathematics and Physics (ZAMP) 37, 859-866, doi:10.1007/BF00953677

[6]. Sadhal S S and Robert E Johnson. (1983). Stokes flow past bubbles and drops partially coated with thin films, Part 1 stagnant cap of surfactant filmexact solution J. Fluid Mech. 126, 237-250.

[7]. Clift, R. (Roland) \& Grace, John R, (joint author.) \& Weber, Martin E, (joint author.) (1978). Bubbles, drops, and particles. Academic Press, New York; London

[8]. Michaelides E E. (2006). Particles Bubbles and Drops: Their motion Heat and Mass Transfer, Singapore: World Scientific.

[9]. Ramana Murthy, J. V., Phani Kumar, M. (2015). Exact solution for flow over a contaminated fluid sphere for Stokes flow, Journal of Physics: Conference Series 662, 012016 doi:10.1088/1742-6596/662/1/012016.

[10]. Dmitry Strunin, Adham Ali. (2019). Rheology and Decay Rate for Frenkel-Biot P1 Waves In Porous Media With Gas Bubbles, International Journal Of Mechanics, Volume 13, pp. 156-163.

[11]. Anh V. Nguyen. (1999). Hydrodynamics of liquid flows around air bubbles in flotation: a review, International Journal of Mineral Processing, Volume 56, Issues 1-4, Pages 165205.

[12]. Stokes V. K. (1971). Effects of couple stresses in fluids on the creeping flow past a sphere, Phy. Fluids, 14, pp. 1580-1582. 
[13]. Stokes V. K. (1984). Theory of fluids with microstructure - An introduction, Springer Verlag.

[14]. Stimson, M., \& Jeffery, G. B. (1926). The Motion of Two Spheres in a Viscous Fluid. Proceedings of the Royal Society A: Mathematical, Physical and Engineering Sciences, 111(757), 110-116.

[15]. T S L Radhika, T Raja Rani, Divy Dwivedi. (2020). Creeping Flow of a Viscous Fluid past a Pair of Porous Separated Spheres, Bulletin of Pure and Applied Sciences. Sect. E Math. Stat. 39E(1), 58-76.

[16]. Happel, J. and Brenner H. (1983). Low Reynolds Number Hydro Dynamics, Martinus Nijho publishers, The Hague.

[17]. Ouadie Koubaiti, Ahmed Elkhalfi, Jaouad ElMekkaoui, Nikos Mastorakis. (2020), Solving the Problem of Constraints Due to Dirichlet Boundary Conditions in the Context of the Mini Element Method, International Journal of
Circuits, Systems and Signal Processing, Volume 14, pp. 12-21.

[18]. Fernando J. P. Caetano, João M. N. A. Fareleira, Carla M. B. P. Oliveira, and William A. (2005). Weakhanded Measurements of the Viscosity of Disobey Phthalate Using Vibrating Wire Technique, J. Chem. Eng. Data, 50, 18751878.

[19]. Kiyofumi Kurihara, Mohamed E. Kandil and Kenneth N. Marsh, Anthony R. H. Goodwin. (2007). Measurement of the Viscosity of Liquid Cyclopentane Obtained with a Vibrating Wire Viscometer at Temperatures between (273 and 353) $\mathrm{K}$ and Pressures below $45 \mathrm{MPa}$, Chem. Eng. Data, 52, 803-807.

[20]. M. Devakar, D. Sreenivasu, B. Shankar. (2014). Analytical solutions of couple stress fluid flow with slip boundary conditions. Alexandria Engineering Journal, 53(3), 723-730.

\section{Appendix- A}

Denote

$$
I_{1}(n)=\int_{-1}^{1} \frac{P_{n}(x)}{(\cos \square \xi-x)^{1 / 2}} d x=\frac{2 \sqrt{2}}{2 n+1} e^{-(n+1 / 2)|\xi|},
$$

$$
\begin{aligned}
& I_{2}(n)=\int_{-1}^{1} \frac{P_{n}(x)}{(\cos \square \xi-x)^{3 / 2}} d x=\frac{2 \sqrt{2}}{\sin \square|\xi|} e^{-(n+1 / 2)|\xi|}, \\
& I_{3}(n)=\int_{-1}^{1} \frac{P_{n}(x)}{(\cos \square \xi-x)^{5 / 2}} d x=\frac{4 \sqrt{2}}{3(\sin \square|\xi|)^{2}}\left(\frac{2 n+1}{2}+\cot \square|\xi|\right) e^{-(n+1 / 2)|\xi|} \\
& I_{4}(n)=\int_{-1}^{1} \frac{\left(1-x^{2}\right) P_{n}^{\prime}(x)}{(\cos \square \xi-x)^{1 / 2}} d x=\frac{n(n+1)}{2 n+1}\left(I_{1}(n-1)-I_{1}(n+1)\right)
\end{aligned}
$$

\section{Appendix- B}

We now derive the expression for the pressure function from equations (5) and (6). Substituting the expressions for the scale factors from (2) in these equations, we get

$$
\begin{aligned}
& \frac{\partial p^{(0)}}{\partial \xi}=-\frac{\mu(\cosh \xi-\tau)}{a} \frac{\partial}{\partial \tau}\left(E^{2} \psi^{(0)}\right), \\
& \frac{\partial p^{(0)}}{\partial \tau}=-\frac{\mu(\cosh \xi-\tau)}{\left(1-\tau^{2}\right)} \frac{\partial}{\partial \xi}\left(E^{2} \psi^{(0)}\right) .
\end{aligned}
$$

Eliminating $\psi^{(0)}$ from these equations, we get

$$
\frac{\partial}{\partial \xi}\left((\cosh \xi-\tau)^{-1} \frac{\partial p^{(0)}}{\partial \xi}\right)+\frac{\partial}{\partial \tau}\left((\cosh \xi-\tau)^{-1}\left(1-\tau^{2}\right) \frac{\partial p^{(0)}}{\partial \tau}\right)=0 .
$$

Using the method of separation of variables, we get

$$
p^{(0)}(\xi, \tau)=(\cosh \xi-\tau)^{1 / 2} \sum_{n=0}^{\infty}\left(H_{n+1} \cosh \left(n+\frac{1}{2}\right) \xi+G_{n+1} \sinh \left(n+\frac{1}{2}\right) \xi\right) P_{n}(\tau),
$$

where's are Legendre's polynomials.

Substituting this and the expression for the stream function in (B.1) and (B.2), the arbitrary constants $H_{n+1}$ and $G_{n+1}$ can be expressed in terms of $A_{n+1}, B_{n+1}, C_{n+1}$ and $D_{n+1}$ [15]. 
Creative Commons Attribution License 4.0

(Attribution 4.0 International, CC BY 4.0)

This article is published under the terms of the Creative

Commons Attribution License 4.0

https://creativecommons.org/licenses/by/4.0/deed.en_US 